\title{
Mesenchymal stromal cells suppress hepatic fibrosis via modulating expression of fibronectin and integrin and inhibiting DNA fragmentation in rats
}

\author{
Sherine M. Ibrahim ${ }^{1}$, Ahmed M. Fayez ${ }^{2}$, Ahmed Maher ${ }^{1}$
}

\author{
${ }^{1}$ Department of Biochemistry, Faculty of Pharmacy, October University for Modern \\ Sciences and Arts, Giza, Egypt \\ 2Department of Pharmacology, Faculty of Pharmacy, October University for Modern \\ Sciences and Arts, Giza, Egypt
}

Submitted: 25 November 2020; Accepted: 24 March 2021

Online publication: 8 April 2021

Arch Med Sci

DOI: https://doi.org/10.5114/aoms/134826

Copyright $\odot 2022$ Termedia \& Banach

\begin{abstract}
Introduction: Liver fibrosis is currently the $11^{\text {th }}$ most common cause of death worldwide. Because of self-renewal, available sources for isolation, and high differentiation properties, multipotent mesenchymal stromal stem cells are suggested to be a potential tool for treatment of liver fibrosis. In this study, we examined the anti-fibrotic and anti-inflammatory effects of bone marrow-derived multipotent mesenchymal stromal stem cells (MSCs) on liver fibrosis induced by carbon tetrachloride on rats relative to silymarin as a standard drug.

Material and methods: This study was performed on 40 male Sprague Dawley rats divided into 4 groups of ten rats each: group 1 served as controls, group 2 served as the $\mathrm{CCl}_{4}$ (diseased) group, group 3 served as the silymarin treated group, and group 4 served as the MSC treated group. Liver fibrosis was assessed by determination of liver markers and fibrogenesis-related genes together with anti-inflammatory markers in the liver tissue. DNA fragmentation was assessed by comet assay.

Results: Mesenchymal stromal stem cells treatment reduced all liver fibrosis markers as well as the oxidative stress and inflammatory markers. Additionally, MSCs reduced the expression of integrins and fibronectin compared with the control group as well as decreasing DNA fragmentation.

Conclusions: Treatment with MSCs significantly ameliorates liver fibrosis in rats. This amelioration was a result of acting on both the anti-inflammatory and anti-fibrotic activity of hepatocytes.
\end{abstract}

Key words: multipotent mesenchymal stromal stem cells, liver fibrosis, fibrogenesis, fibronectin, integrin- $\beta 1$.

\section{Introduction}

Epidemiological data show that liver diseases have gradually become an increasing health burden worldwide contributing to 2 million of the world's deaths every year [1]. Hepatic fibrosis is characterized by activation of hepatic stellate cells (HSC) and accumulation of extracellular matrix (ECM) proteins inside the liver parenchyma, leading to chronic injury and eventually loss of liver functions [2]. Over the last decades, the gold-standard treatment of liver cirrhosis was orthotopic liver transplantation. Although considered as a substitution that can promote liver

\author{
Corresponding author: \\ Sherine M. Ibrahim \\ Department of Biochemistry, \\ Faculty of Pharmacy \\ October University for \\ Modern Sciences and Arts \\ Giza, Egypt \\ E-mail: sherinemahmoud@ \\ msa.eun.eg
}


function, this treatment is limited due to the scarcity of donors, the cost of the operation, and the need for lifelong immunosuppression [3-5].

There is an emerging role for multipotent mesenchymal stromal stem cells (MSCs) in regenerative medicine due to their high proliferation rate [6-9], their ability to produce several active molecules that are capable of inhibiting inflammation and stimulating the recovery of injured cells, and their capability to differentiate into HSCs with the successful expression of hepatic specific markers. Recently, many studies have shown that MSCs injected in rat models have anti-inflammatory and anti-fibrotic functions improving liver damage [6].

A known model for cirrhosis and liver damage is the $\mathrm{CCl}_{4}$ model, where $\mathrm{CCl}_{4}$ is metabolized inside the body by CYP2E1 to release highly reactive free radicles. These reactive free radicals interact with different cellular macromolecules, especially membrane lipids, forming lipid peroxides [10]. This study highlights recent findings to assess the therapeutic effects of MSCs in the treatment of liver fibrosis in a rat model of liver fibrosis induced by $\mathrm{CCl}_{4}$ by evaluating changes in liver histopathology, liver function markers, fibronectin and integrin- $\beta 1$ gene expression, and DNA integrity in comparison to silymarin as a standard drug.

\section{Material and methods}

\section{Animals}

Sprague Dawley male albino rats weighing 180-220 g were purchased from the Nile Center of Experimental Research, Mansoura, Egypt. The animals were maintained at constant temperature $\left(22^{\circ} \mathrm{C}\right)$. Food and water were given ad libitum throughout the experimental period. The study was approved by the Ethical Committee of the MSA (Bp7/EC7/2018F).

\section{Experimental design}

The animals were randomly divided into four groups $(n=10)$ as follows:

Group 1: served as the control group receiving olive oil ( $2 \mathrm{ml} / \mathrm{kg}$ single oral dose) twice per week for 8 consecutive weeks.

Groups 2: served as a positive control (liver fibrosis). The rats received orally $2 \mathrm{ml} / \mathrm{kg} \mathrm{CCl}_{4}(20 \%$ olive oil dilution) twice per week for 8 consecutive weeks for the induction of liver fibrosis [11]. $\mathrm{CCl}_{4}$ was used in this study for liver fibrosis induction because this model closely resemble that of humans [12].

Group 3: served as a standard treatment group receiving $100 \mathrm{mg} / \mathrm{kg}$ silymarin (Sigma Aldrich \#S0292) orally daily along with $2 \mathrm{ml} / \mathrm{kg} \mathrm{CCl}_{4}(20 \%$ olive oil dilution) orally twice per week for 8 consecutive weeks.
Group 4: served as an MSC-treated group (MSC group). These were treated with $1 \times 10^{6} \mathrm{MSC}$ suspended in $0.5 \mathrm{ml}$ of PBS, single-dose i.v. along with $2 \mathrm{ml} / \mathrm{kg} \mathrm{CCl}_{4}$ (20\% olive oil dilution) orally twice per week for 8 consecutive weeks. Mesenchymal stromal stem cells were injected 4 weeks after starting the $\mathrm{CCl}_{4}$ administration [13].

\section{Mesenchymal stromal stem cells isolation and identification}

Six-week-old Sprague Dawley male albino rats were euthanized with thiopental. Mesenchymal stromal stem cells were isolated from the bone marrow of the tibiae and femurs after being carefully dissected [14]. Bone marrow was carefully flushed with Dulbecco's modified Eagle's medium. Nucleated cells were completely isolated by a gradient (Ficoll-Paque from Pharmacia) and then suspended in $1 \%$ penicillin-streptomycin culture (Sigma-Aldrich, St. Louis, MO, USA). The isolated cells were cultured for $12-14$ days at $37^{\circ} \mathrm{C}$ and $5 \%$ humidified $\mathrm{CO}_{2}$. Cells are then harvested by trypsinization and incubated in a culture flask.

\section{Assessment of liver fibrosis}

Liver function tests: Serum aspartate aminotransferase (AST) and alanine aminotransferase (ALT) were assayed using (Biodiagnostics, (A, USA) and serum albumin was assayed using a QuantiChrom BCG Albumin Assay Kit (Bioassay Systems).

Determinations of oxidative stress and inflammation markers: The liver was weighed and a fraction was homogenized in ice-cold phosphate-buffered saline (PBS) to prepare a $20 \%$ solution. Lipid peroxidation marker expressed as malondialdehyde (MDA) and glutathione (GSH) as an antioxidant marker were assayed using the dithio-binitrobenzoic acid method [15] and the thiobarbituric acid reactive substances (TBARS) method [16].

For the anti-inflammatory markers, a fraction of the liver was carefully homogenized in a hypotonic lysis buffer using a protease inhibitor. Interleukin-10 (IL-10), an anti-inflammatory marker, and tumor necrosis factor (TNF- $\alpha$ ), a pro-inflammatory marker, were determined using ELISA kits provided by MyBiosource, Inc. (San Diego., USA)

Expression of fibronectin and integrins- $\beta 1$ : RNA was extracted for examination from the liver according to the manufacturer's instructions using an RNA Extraction Kit (iNtrON Biotechnology, Korea). Fibrotic gene expression for fibronectin and integrin- $\beta 1$ was determined using quantitative reverse-transcription polymerase chain reaction (RTPCR) (Brilliant II QRT-PCR Master Mix Kit, 1-Step, Agilent, CA, USA) using the following primers (Table I). 
Table I. Expression of $\beta$-actin, fibronectin and integrin- $\beta 1$

\begin{tabular}{|lcc|}
\hline$\beta$-Actin & F: TCCTCCTGAGCGCAAGTACTCT & V01217.1 \\
& R: GCTCAGTAACAGTCCGCCTAGAA & \\
\hline Fibronectin & $\begin{array}{l}\text { F: ATGTGGACCCCTCCTGATAGT } \\
\text { R: GCCCAGTGATTTCAGCAAAGG }\end{array}$ & NM_001276408.1 \\
\hline Integrin- $\beta 1$ & $\begin{array}{l}\text { F: GCCAGGGCTGGTTATACAGA } \\
\text { R: TCACAATGGCACACAGGTTT }\end{array}$ & XM_011248315.1 \\
\hline
\end{tabular}

Results were compared with those of the control group using the $2^{-\Delta \Delta C t}$ method.

\section{Western blotting}

The ReadyPrep protein extraction kit, Bio-Rad Inc (Catalog \#163-2086), was employed according to the manufacturer's instructions to the homogenized liver tissues. The Bradford Protein Assay Kit (SK3041) provided by Bio basic Inc (Markham Ontario L3R 8T4 Canada) was used for protein quantification in all samples. $20 \mu \mathrm{g}$ of protein of each sample was then loaded with an equal volume of $2 \times$ Laemmli sample buffer and separated using polyacrylamide gel electrophoresis. The blot was then run for $7 \mathrm{~min}$ at $25 \mathrm{~V}$ to allow transfer of protein bands from the gel to a PVDF membrane using Bio-Rad Trans-Blot Turbo. Fibronectin (catalog NBP1-84468, Novus Biologicals) and $\beta 1$-integrin (catalog\# 4706, Cell Signaling Technology, USA) primary antibodies were incubated overnight against the blotted target protein at $4^{\circ} \mathrm{C}$. Finally, incubation was done in the HRP-conjugated secondary antibody (goat anti-rabbit IgG - HRP; $1 \mathrm{mg}$ goat mab - Novus Biologicals) solution against the blotted target protein for $1 \mathrm{~h}$ at room temperature. A chemiluminescent substrate (Clarity Western ECL substrate Bio-Rad cat\#170-5060) was applied to the blot. Image analysis software was used to read the band intensity of the target proteins against $\beta$-actin by protein normalization on the ChemiDoc MP imager.

\section{Histopathological assessment}

Hematoxylin and eosin staining: After fixation of liver specimens in $10 \%$ formaldehyde in PBS, liver tissues were dehydrated, embedded in paraffin and sections were made at a thickness of $5 \mu \mathrm{m}$. These sections were carefully stained with hematoxylin and eosin (H \& E) for histopathology [17].

METAVIR scoring system for fibrosis assessment: The hepatic fibrosis was determined using the METAVIR scoring system [17]. The fibrosis score is determined using a five-point scale ( 0 = no fibrosis, $\mathrm{F} 1=$ portal fibrosis without septa, F2 = few septa, F3 = numerous septa without cirrhosis, F4 = cirrhosis).

\section{Immunohistochemistry}

Briefly, liver tissue sections were cut into $5 \mu \mathrm{m}$ sections and subjected to deparaffinization, dehy- dration and heat-induced antigen retrieval then endogenous peroxidase and protein blocking steps. After washing, liver tissue sections were incubated with primary antibodies (anti-CD68, Santa Cruz Biotechnology Inc.) overnight at $4^{\circ} \mathrm{C}$ in a humid chamber with a dilution ratio of $1: 200$ in PBS. HRP-labelled secondary antibodies (Abcam, UK) were applied for 2 hours after washing. Finally, a DAB-substrate kit was used for color development and Mayer's hematoxylin was used as a counter stain. Negative control slides were obtained by escaping the primary antibody step. Positive expression was quantified as area percent (\%).

\section{Comet assay}

The extent of DNA damage was accessed using the comet assay under alkaline conditions. Comet tail length was measured first by fluorescence microscopy and then it was analyzed using the Casplab comet assay Software v1.2.3 (Tritek Corporation, Summerduck, VA). From each group, ten cells were analyzed.

\section{Statistical analysis}

All data are expressed as mean \pm SD. The difference between all groups was determined by GraphPad Prism 6, using the one-way ANOVA test and Tukey's Kramer multiple comparison test with significance at the $p$-value $<0.05$.

\section{Results}

\section{Mesenchymal stromal stem cells improved} liver function tests

After induction of liver fibrosis with $\mathrm{CCl}_{4}$, both ALT (U/I) and AST (U/l) levels were increased by 1.7- and 2.5-fold in the serum while the albumin concentration decreased. Silymarin administration enhanced the liver function tests and decreased the level of both enzymes as well as enhancing the synthesis of albumin. On comparing the MSC group with silymarin, although both decreased the liver enzymes and increased the albumin synthesis, MSCs were able to reduce the more liver-specific ALT significantly more than silymarin (Table II), indicating a higher healing power. 
Table II. Effect of MSC therapy on liver function enzymes and albumin in the studied groups

\begin{tabular}{|lccc|}
\hline Group & ALT [U/l] & AST [U/l] & Albumin [g/dl] \\
\hline Control & $37.59 \pm 3.56$ & $38.42 \pm 3.59$ & $3.91 \pm 0.18$ \\
\hline $\mathrm{CCl}_{4}$ & $75.38^{\mathrm{a}} \pm 5.28$ & $145.1^{\mathrm{a}} \pm 16.12$ & $1.9^{\mathrm{a}} \pm 0.24$ \\
\hline Silymarin & $53.8^{\mathrm{a}, \mathrm{b}} \pm 3.89$ & $57.11^{\mathrm{a}, \mathrm{b}} \pm 4.49$ & $3.18^{\mathrm{b}} \pm 0.28$ \\
\hline MSCs & $47.12^{\mathrm{a}, \mathrm{b}, \mathrm{c}} \pm 5.09$ & $49.54^{\mathrm{a}, \mathrm{b}} \pm 3.43$ & $3.27^{\mathrm{b}} \pm 0.25$ \\
\hline
\end{tabular}

Each value represents the mean of 10 experiments \pm SD. Statistical analysis was performed using one-way ANOVA followed by Tukey's

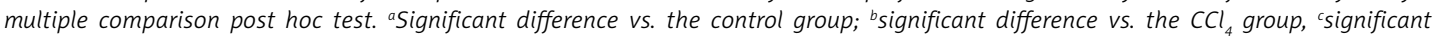
difference vs. the silymarin group.

MSC - mesenchymal stromal stem cells, AST - aspartate aminotransferase, ALT - alanine aminotransferase.

\section{Mesenchymal stromal stem cells possess antioxidative and anti-inflammatory properties}

The $\mathrm{CCl}_{4}$ resulted in a 2 -fold increase in the level of MDA and a nearly 3-fold decline in the level of GSH compared with control rats, indicating liver damage. Treatment with silymarin decreased MDA by around $30 \%$ and enhanced GSH by more than $50 \%$. Groups treated with MSCs showed MDA levels comparable to the silymarin group and $26 \%$ higher levels of GSH (Figure 1).

The anti-inflammatory properties of silymarin and MSCs were assessed by measuring the IL-10 and TNF- $\alpha$ levels. Induction of fibrosis with $\mathrm{CCl}_{4}$ resulted in a decrease of IL-10 and an increase of proinflammatory TNF- $\alpha$. Upon treatment with silymarin, IL-10 increased by more than 1.8-fold while MSCs increased it by 2.6-fold. On the other hand, silymarin decreased TNF- $\alpha$ by $25 \%$ compared to the diseased group while MSCs surpassed this to reach a $56 \%$ decrease in the levels of TNF- $\alpha$ (Figure 2). MSCs showed both anti-inflammatory and antioxidative stress properties.

A

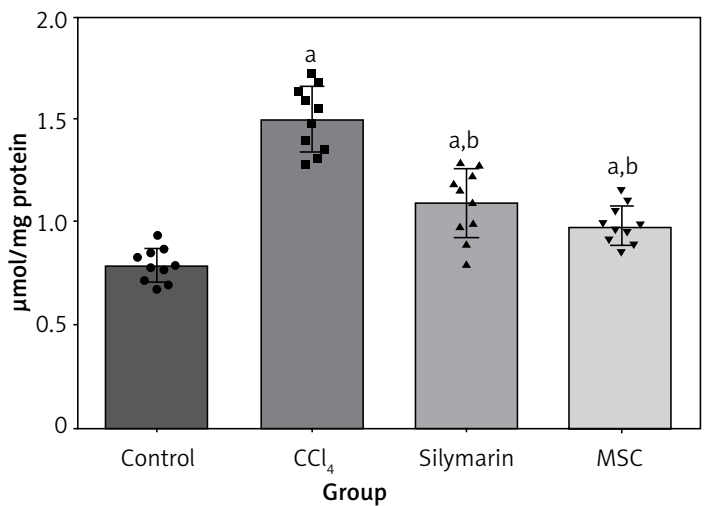

\section{Mesenchymal stromal stem cells suppresses the expression of fibrogenesis- related genes}

QRT-PCR revealed a more than 2.5-fold increase in both fibronectin and integrin- $\beta 1$ expression in rats after administering $\mathrm{CCl}_{4}$ for 8 weeks, showing a role for both proteins in the progression of fibrosis. These values were decreased after treatment. Silymarin decreased the expression of fibronectin by $74 \%$ and integrin- $\beta 1$ by $77 \%$. Treatment with MSCs lowered the expression of both proteins to the levels of the control group where fibronectin decreased by $32 \%$ more and integrin- $\beta 1$ by $40 \%$ compared to the standard silymarin group (Figure 3 ).

\section{Western blotting analysis}

The results of QRT-PCR were confirmed with the western blotting analysis as the expression of both genes was overexpressed in cases of fibrosis and treatment decreased their expression levels. Treatment with MSCs shows a greater decrease in the level of both fibronectin and integrin $\beta 1$ (Figure 4).

\section{B}

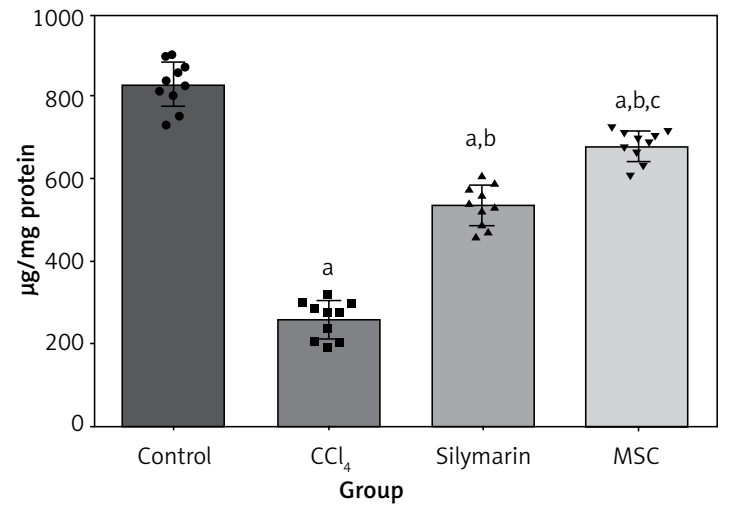

Figure 1. A - Effect of therapy on MDA levels in the studied groups. Treatment decreased liver peroxidation compared to the diseased group. There was no significant difference between silymarin and MSCs. B - Effect of therapy on GSH levels in the studied groups. Both treatments enhanced GSH synthesis with the MSC group showing better antioxidant results

Each value represents the mean of 10 experiments \pm SD. ${ }^{a}$ Significant difference vs. the control group, ${ }^{b}$ significant difference vs. the $\mathrm{CCl}_{4}$ group, ${ }^{c}$ significant difference vs. the silymarin group. MSC - mesenchymal stromal stem cells. 
A

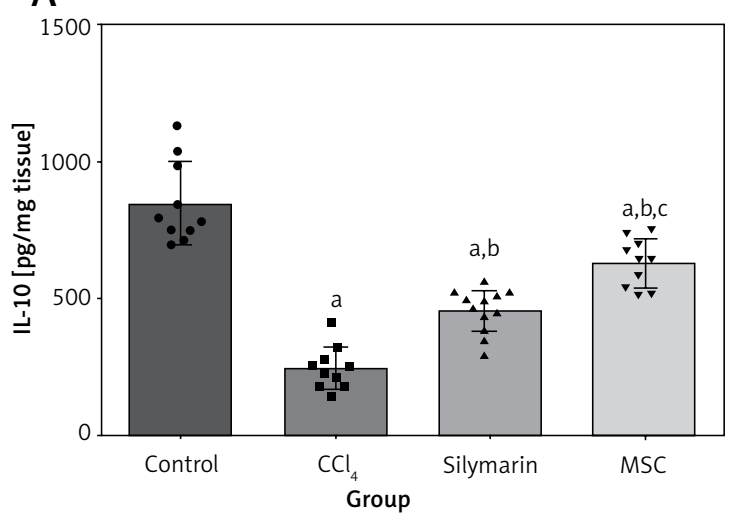

B

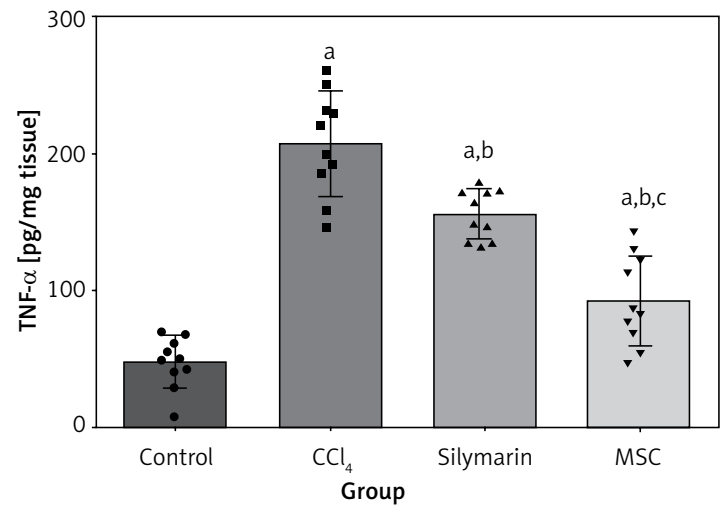

Figure 2. A - Effect of therapy on IL-10 levels in the studied groups. Treatment increased expression of the antiinflammatory cytokine compared to the diseased group. Expression of IL-10 in the MSC group was significantly higher than that of the silymarin group. B - Effect of therapy on the TNF- $\alpha$ levels in the studied groups. Both treatments decreased TNF- $\alpha$ levels, showing anti-inflammatory properties. MSCs were 17 -fold more potent than silymarin Each value represents the mean of 10 experiments \pm SD. ${ }^{a}$ Significant difference vs. the control group, ${ }^{b}$ significant difference vs. the $\mathrm{CCl}_{4}$ group, 'significant difference vs. the silymarin group. MSC - mesenchymal stromal stem cells.

A

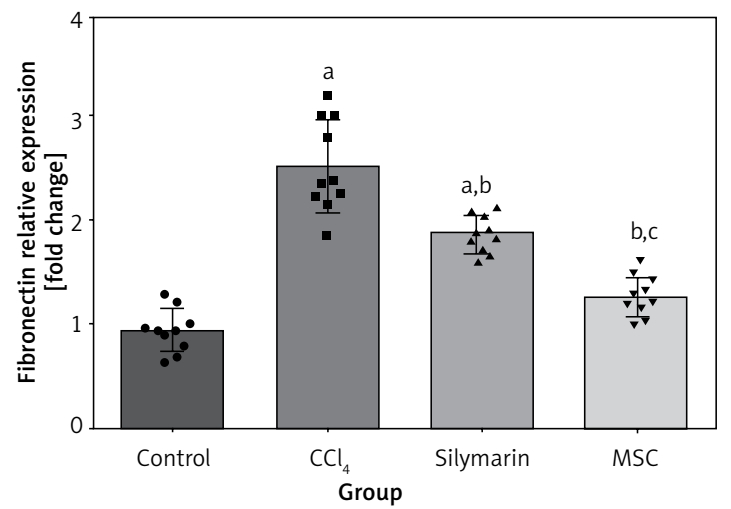

B

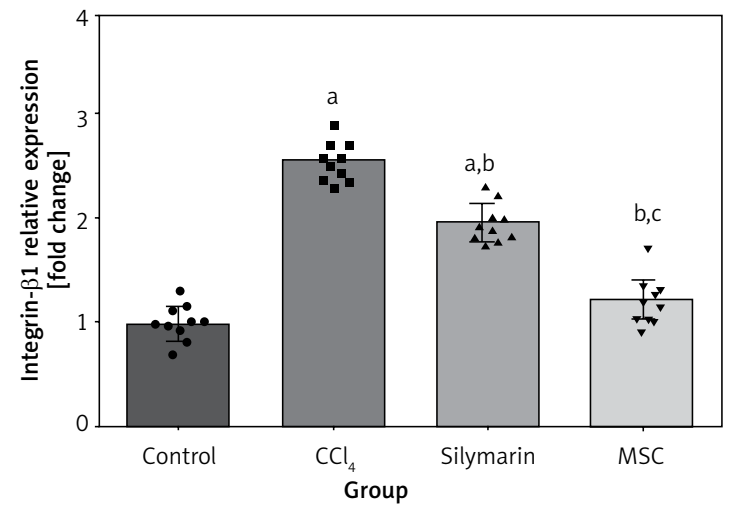

Figure 3. A - Effect of therapy on expression levels of fibronectin in the studied groups. Fibronectin expression was enhanced by the administration of $\mathrm{CCl}_{4}$ and treatment decreased its expression compared to the diseased group. MSC treatment decreased the expression level to that of the control group. B - Effect of therapy on expression levels of integrin- $\beta 1$ in the studied groups. Integrin- $\beta 1$ expression was enhanced by $\mathrm{CCl}_{4}$. Treatment with silymarin significantly decreased its expression but MSC treatment decreased the expression level to that of the control group

Each value represents the mean of 10 experiments $\pm S D$. ${ }^{a}$ Significant difference vs. the control group, ${ }^{b}$ significant difference vs. the $\mathrm{CCl}_{4}$ group, 'significant difference vs. the silymarin group. MSC - mesenchymal stromal stem cells.

\section{Histopathological assessment shows improvement after treatment with MSCs}

Mesenchymal stromal stem cells decrease liver fibrosis: $\mathrm{H}$ \& $\mathrm{E}$ staining revealed significant changes obtained in the histological examination between the control, untreated and treated groups. In the $\mathrm{CCl}_{4}$ group, the liver tissue shows activation of Kupffer cells and sporadic hepatocytes necrosis while both the silymarin and the MSC groups showed slight activation of Kupffer cells and few necrotic effects of hepatocytes (Figure 5).

Mesenchymal stromal stem cells reduces fibrosis in the METAVIR scoring system: The fibrosis score was assessed using the METAVIR scoring system. Both silymarin and MSCs had better scores than the diseased groups but MSCs had better results regarding the extent of fibrosis (Table III). Both the H\&E staining and the METAVIR Scoring showed a positive impact of the MSCS on the extent of liver fibrosis.

\section{Immunohistochemistry}

Immune expression of CD68 in liver tissue is illustrated in Figure 6. The control group showed normal limited expression of CD68 cells. In contrast, CD68 expression was significantly increased in the $\mathrm{CCl}_{4}$ group compared to the control group. The silymarin treated group exhibited slight improvement in comparison to the $\mathrm{CCl}_{4}$ group. The MSC treated grouped showed the greatest reduction in CD68 positive cells compared with the $\mathrm{CCl}_{4}$ group (Figure 6). 


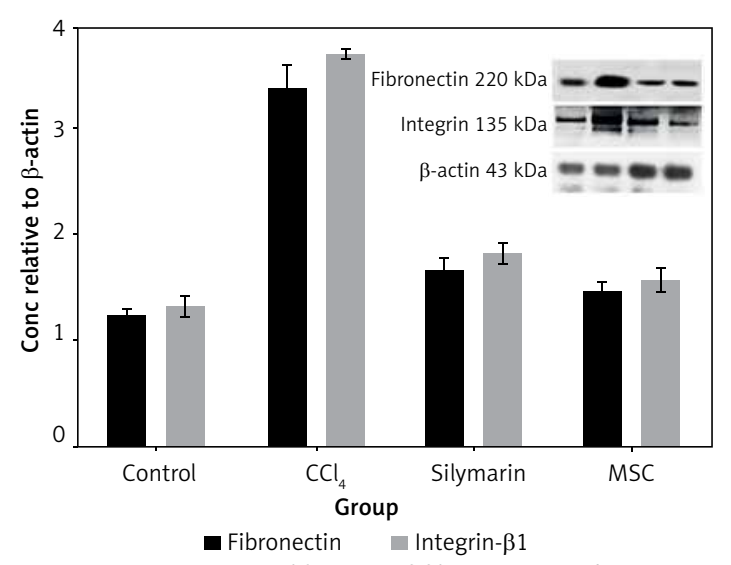

Figure 4. Western blotting of fibronectin and integrin. Administration of $\mathrm{CCl}_{4}$ enhanced the expression of both fibronectin and integrin by more than 2.5 -fold compared to the control group. Treatment with silymarin or MSCs resulted in a decrease in the levels of both genes by around 1.9-fold with silymarin and 2.2-fold with MSCs

MSC - mesenchymal stromal stem cells.

\section{Mesenchymal stromal stem cells inhibits DNA fragmentation}

The effect of administration of either $\mathrm{CCl}_{4}$ or $\mathrm{CCl}_{4}$ followed by treatment with silymarin or MSCS on liver DNA is shown in Figure 7. A significant more than 8 -fold increase in the tail length and 3.7-fold increase in tail DNA\% (tDNA\%) was observed in the liver tissues of rats intoxicated with $\mathrm{CCl}_{4}$. Treatment with silymarin and MSCs significantly protected the rats' livers from DNA damage as indicated by a decrease in tail length $\%$ and tDNA\%. Silymarin decreased the tail length\% by $24 \%$ and the tDNA\% by $40 \%$ compared to the diseased group while MSCs restored both to the level of the control group as it decreased the length by $20 \%$ and the tDNA\% by $35 \%$ more than the silymarin (Figure 7). The results show that the MSCs have a great protective effect on the integrity of the DNA.

\section{Discussion}

Recent studies have focused on the role of using MSCs in medicine for the treatment of multiple diseases such as Alzheimer's, lung fibrosis, corneal diseases, and digestive diseases [18-22]. In this study, injection of MSCs in rats with liver fibrosis resulted in improving liver functions and decreasing the extent of fibrosis, inhibiting the expression of fibrogenic genes and decreasing hepatic DNA fragmentation.

The blood samples taken from the tail were used to assess the extent of liver damage using ALT, AST, and serum albumin, and the damage was confirmed. Treatment with MSCs gradually attenu-
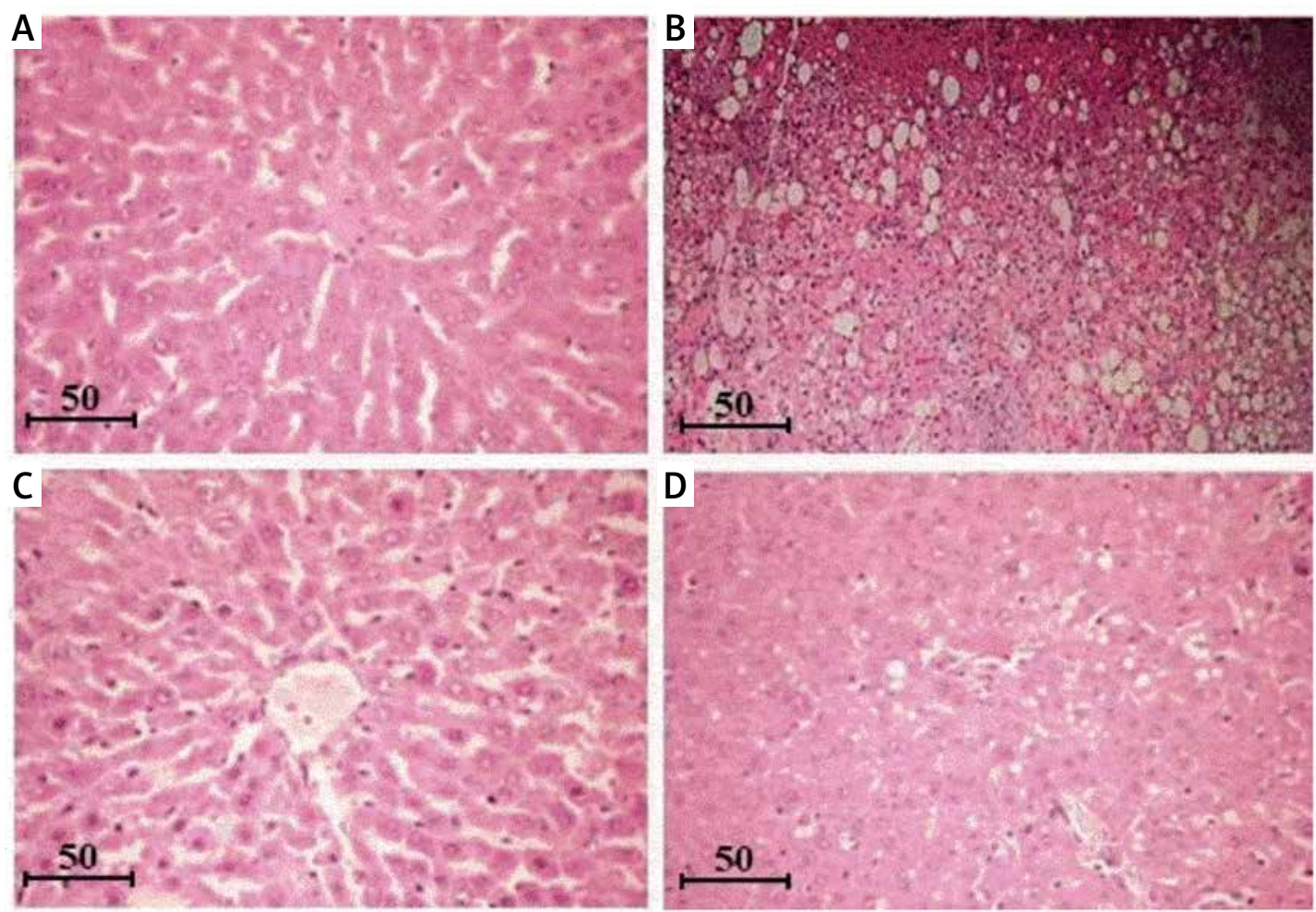

Figure 5. A - Liver of control rat showing no histopathological changes. B - Liver of $\mathrm{CCl}_{4}$ intoxicated rat showing Kupffer cells' activation and necrosis of sporadic hepatocytes. C - Liver of $\mathrm{CCl}_{4}$ intoxicated rat treated with silymarin showing some Kupffer cells' activation and decreased necrosis of sporadic hepatocytes. D - Liver of $\mathrm{CCl}_{4}$ intoxicated rat treated with MSCs showing slight Kupffer cells' activation and decreased necrosis of sporadic hepatocytes. H \& E stain $(\times 400)$ 
Table III. The fibrosis score was assessed on a five-point scale using the METAVIR scoring system

\begin{tabular}{|lcccc|}
\hline Fibrous score & Normal & $\mathrm{CCl}_{4}$ & Silymarin & MSCs \\
\hline F0 & 9 & 0 & 0 & 2 \\
\hline F1 & 1 & 2 & 4 & 6 \\
\hline F2 & 0 & 5 & 5 & 0 \\
\hline F3 & 0 & 2 & 1 & 0 \\
\hline F4 & 0 & 1 & 0 & 0 \\
\hline
\end{tabular}

The fibrosis score was assessed on a five-point scale using the METAVIR scoring system where FO= no fibrosis, F1 = portal fibrosis without septa, F2 = few septa, F3= numerous septa without cirrhosis, F4 = cirrhosis. MSC - mesenchymal stromal stem cells.
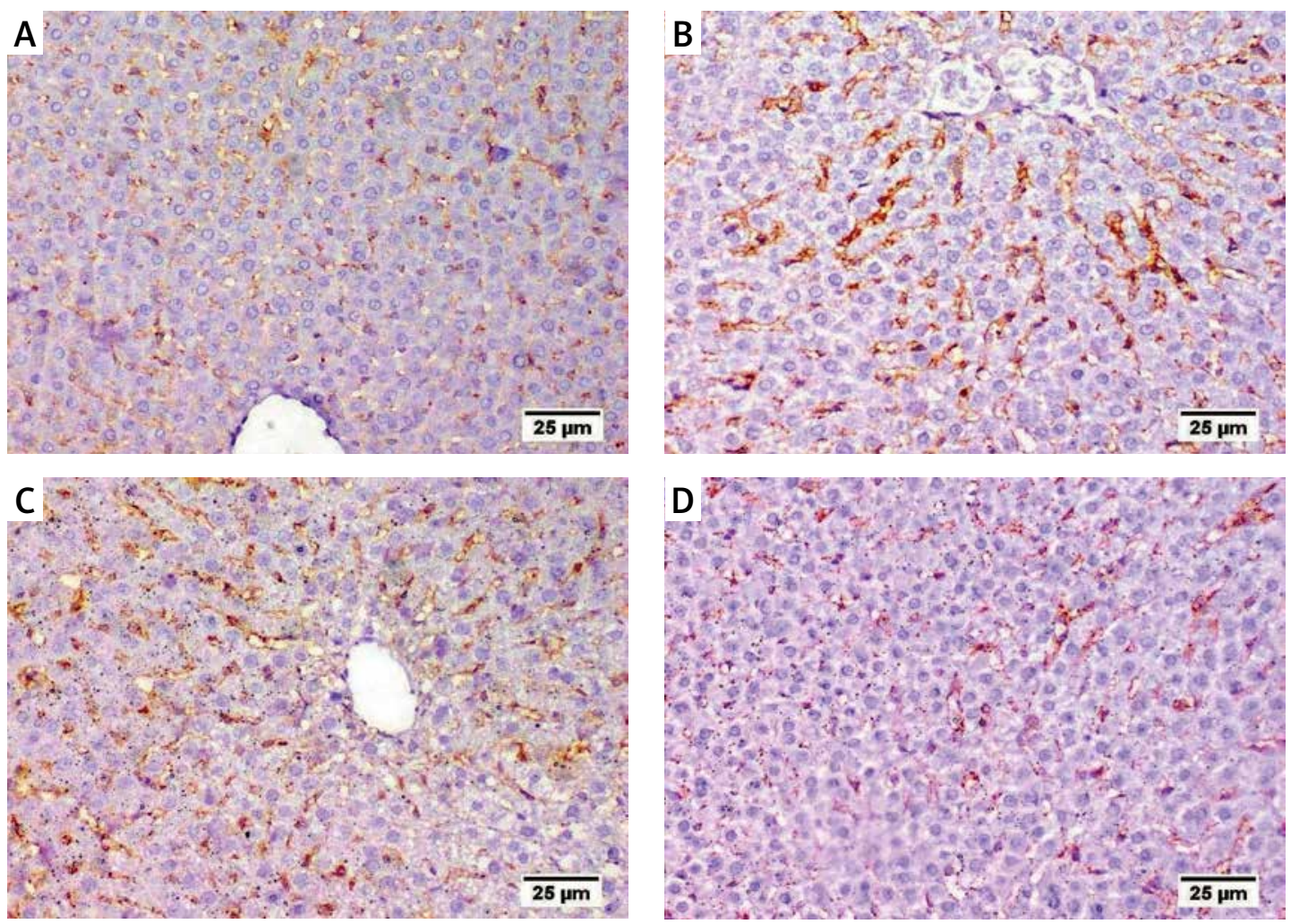

$\mathrm{E}$

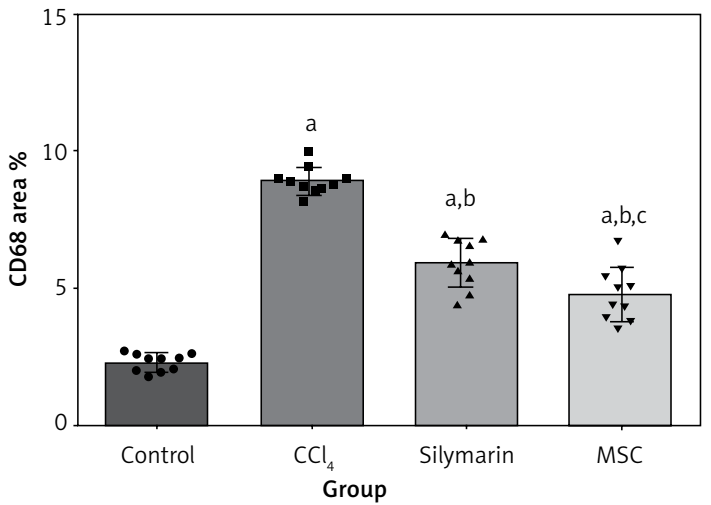

ated the liver injury by reducing the $\mathrm{CCl}_{4}$-elevated serum levels of AST and ALT more than the control, diseased, and silymarin groups. The level of albumin was elevated in the MSC group significantly more than the silymarin group. Previous studies agreed with our study and showed elevated lev-

Figure 6. Immunostaining of CD68 in liver tissue, A - control group, B - $\mathrm{CCl}_{4}$ group, C - silymarin treated group and D - MSC treated group. E - Quantification of CD68 positive cells as area \% of expression

Values are presented as means \pm SE. Differences were considered significant at $p<0.05$. 'a Significant difference vs. the control group, bsignificant difference vs. the $\mathrm{CCl}_{4}$ group, 'significant difference vs. the silymarin group. MSC - mesenchymal stromal stem cells.

els of albumin and reduced liver damage markers, showing the ability of MSCs to restore liver functions [23]. Furthermore, the decrease in liver enzymes was accompanied by a similar decrease in the levels of TNF- $\alpha$ in the MSC group compared to other groups [24]. The improvement in the MSC 
A
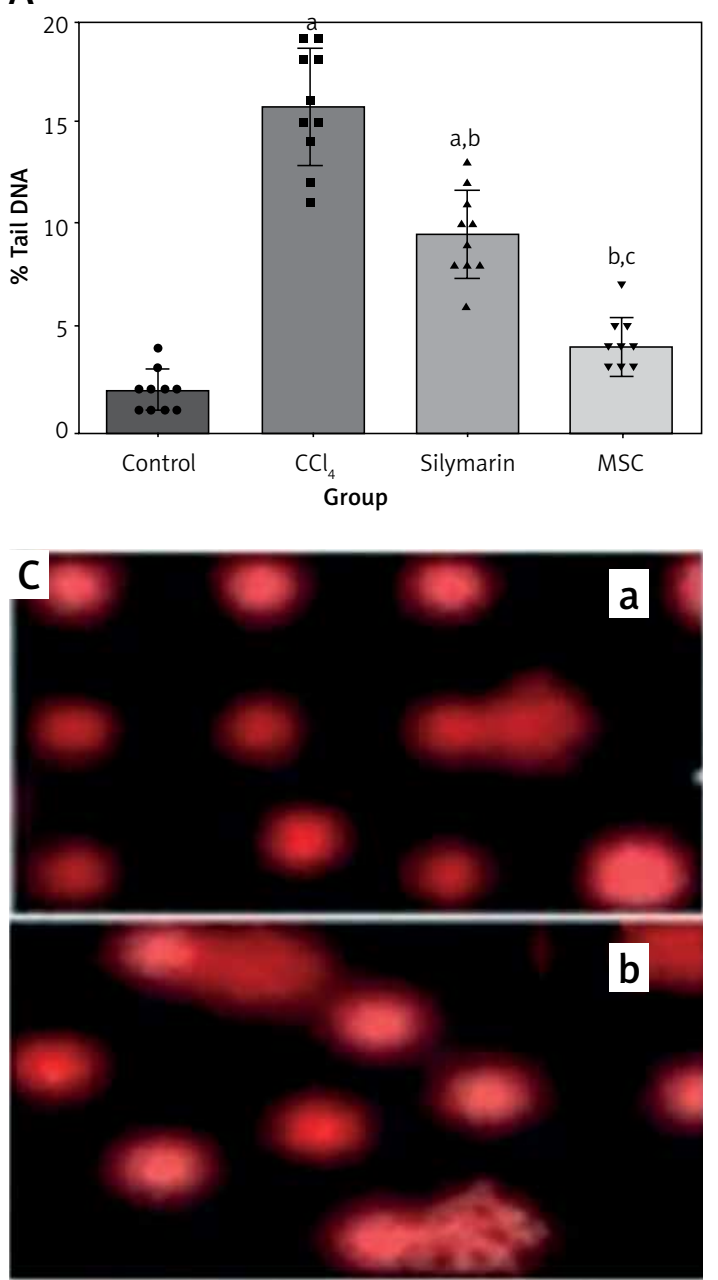

B
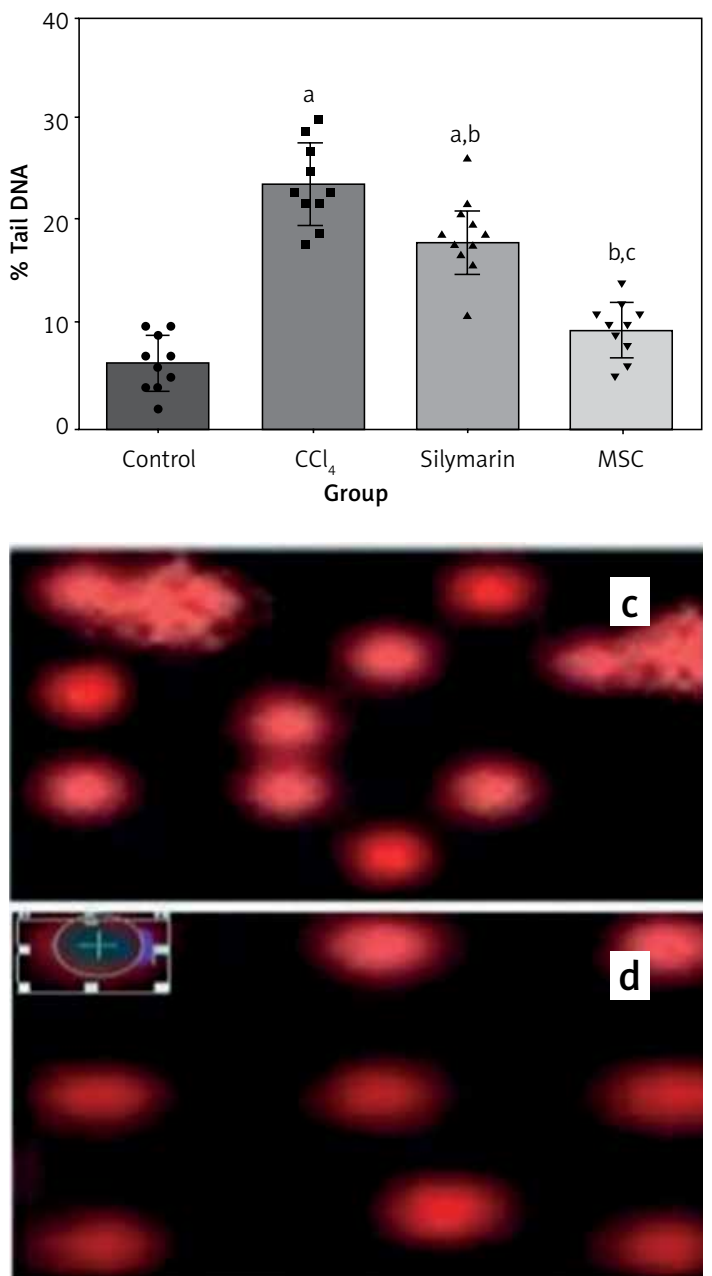

Figure 7. Effect of treatments on integrity of hepatocytes' DNA. A - \% tail DNA. Both minimized DNA degradation but MSCs were 2.4-fold more protective. B - \% tail length. Both minimized DNA degradation but MSCs were 1.9fold more protective. C - Comets from hepatocytes, a: control, b: $\mathrm{CCl}_{4}$, c: silymarin treated, and d: MSC treated Each value represents the mean of 10 experiments \pm SD. ${ }^{a}$ Significant difference vs. the control group, ${ }^{b}$ significant difference vs. the $\mathrm{CCl}_{4}$ group, 'significant difference vs. the silymarin group. MSC - mesenchymal stromal stem cells.

group can be attributed to MSCs' ability to regenerate the liver cells after $\mathrm{CCl}_{4}$ injury [7].

Additionally, our work shows that injection with MSCs ameliorates the levels of GSH and reduces the MDA level as well within the MSC group compared to other groups, indicating its ability to reduce oxidative stress caused by $\mathrm{CCl}_{4}$ We believe that MSCs' soluble mediators could play a role in reducing inflammation and oxidative stress, which may in turn play a role in liver fibrosis [25-27]. Similar results were confirmed by other studies $[24,28]$. MSCs are suggested to have an essential anti-inflammatory and immunomodulatory role that allows them to have a very important role in liver recovery [28].

Liver fibrosis is characterized by the accumulation of extracellular matrix proteins in the injured liver tissue where hepatic stellate cells (HSC) play an important role. Upon injury, the level of trans- forming growth factor-beta (TGF- $\beta$ ) increases and results in the activation of the HSC through the TGF- $\beta$ /SMAD pathway $[29,30]$. This activation results in the production of fibronectin, an ECM protein and a major profibrotic factor [29]. In the present study, MSC treatment decreased the level of fibronectin expression compared to the silymarin group through a dual pathway. First, MSCs decrease the proliferation of HSC and promotes its apoptosis [31]. The second is through the elevation of IL-10, which in turn downregulates TGF- $\beta$ and inhibits the transcription of fibronectin [32, 33]. MSCs also successfully inhibited the expression of integrin, a potent activator of TGF- $\beta[34,35]$. The decrease in the levels of both fibronectin and integrin can explain the decrease in the extent of fibrosis and the antifibrotic effect of MSCs. This also explains the reduction in DNA damage within the liver tissue as assessed by the comet assay. 
The histopathological examination is the main tool for the diagnosis of liver diseases. In our study, MSC groups showed slight activation of Kupffer cells and sparse necrosis of hepatocytes compared to other groups by its regenerative properties. Also, we assessed stages of fibrosis through the METAVIR scoring system. According to this system, a significant improvement of hepatic fibrosis was observed in the MSC group compared to diseased and silymarin groups. Our findings were confirmed by previous studies which also indicated that MSCs inhibit production of the profibrotic factors fibronectin and integrin, as was observed in our study [36, 37].

A study by Popp et al., in 2007 [38], suggested that MSCs do not contribute to liver regeneration, but we believe that there were 2 reasons for this. The first is that the authors used dipeptidyl peptidase IV as a marker for hepatic regeneration which, as the authors stated, is a very late marker of hepatic development, and the second is that part of the action of MSCs is mediated through soluble mediators and not only differentiation $[26,27]$.

Our results showed that MSCs have more regenerative, anti-fibrotic, and anti-inflammatory properties than silymarin, which is a standard drug in the treatment of liver fibrosis. Also our findings were strongly proved by the histopathological evaluation, the METAVIR scoring system, and comet assay. MSCs can restore liver function and structure through several mechanisms which include secretion of many growth factors and cytokines that help to reduce inflammation and fibrogenesis, and repair the injured hepatic cells [39].

In conclusions, MSCs treatment ameliorates all liver functions in this rat model of $\mathrm{CCl}_{4}$-induced liver fibrosis, which was clearly shown by histopathological assessment and comet assay. Furthermore, MSC treatment decreased the expression of fibrogenesis and inflammation and restored the liver function significantly more than silymarin treatment. Considering safety, MSC transfusion is a potential tool for treatment of liver fibrosis.

\section{Acknowledgments}

All authors wish to express their sincere thanks to all staff of the animal house in the Modern Sciences and Arts University for their cooperation in carrying out this research. We are also grateful to the laboratory technicians for their contribution.

\section{Conflict of interest}

The authors declare no conflict of interest.

\section{References}

1. Asrani SK, Devarbhavi H, Eaton J, Kamath PS. Burden of liver diseases in the world. J Hepatol 2019; 70: 151-71.
2. Schuppan D, Ashfaq-Khan M, Yang AT, Kim YO. Liver fibrosis: direct antifibrotic agents and targeted therapies. Matrix Biol 2018; 68-69: 435-51.

3. Kumar A, Tripathi A, Jain S. Extracorporeal bioartificial liver for treating acute liver diseases. J Extra Corpor Technol 2011; 43: 195-206.

4. Mendizabal M, Silva MO. Liver transplantation in acute liver failure: a challenging scenario. World J Gastroenterol 2016; 22: 1523-31.

5. Ferreira Meirelles R Jr, Salvalaggio P, de Rezende MB et al. Liver transplantation: history, outcomes and perspectives. Einstein (Sao Paulo) 2015; 13: 149-52.

6. Doyle EC, Wragg NM, Wilson SL. Intraarticular injection of bone marrow-derived mesenchymal stem cells enhances regeneration in knee osteoarthritis. Knee Surg Sports Traumatol Arthrosc 2020; 28: 3827-42.

7. Zhang L, Dong Z, Zhang J. Immunomodulatory role of mesenchymal stem cells in Alzheimer's disease. Life Sci 2020; 246: 117405.

8. Ocansey DKW, Pei B, Yan Y, et al. Improved therapeutics of modified mesenchymal stem cells: an update. J Transl Med 2020; 18: 42.

9. Nacher-Soler G, Garrido JM, Rodríguez-Serrano F. Hearing regeneration and regenerative medicine: present and future approaches. Arch Med Sci 2019; 15: 957-67.

10. Manibusan MK, Odin M, Eastmond DA. Postulated carbon tetrachloride mode of action: a review. J Environ Sci Health C Environ Carcinog Ecotoxicol Rev 2007; 25: 185-209.

11. Gan D, Zhang W, Huang C, et al. Ursolic acid ameliorates CCl4-induced liver fibrosis through the NOXs/ROS pathway. I Cell Physiol 2018; 233: 6799-813.

12. Iredale JP, Thompson A, Henderson NC. Extracellular matrix degradation in liver fibrosis: biochemistry and regulation. Biochim Biophys Acta 2013; 1832: 876-83.

13. Truong NH, Nguyen NH, Van Le T, et al. Comparison of the treatment efficiency of bone marrow-derived mesenchymal stem cell transplantation via tail and portal veins in CCl4-induced mouse liver fibrosis. Stem Cells Int 2016; 2016: 5720413

14. Alhadlaq A, Mao JJ. Mesenchymal stem cells: isolation and therapeutics. Stem Cells Dev 2004; 13: 436-48.

15. Rotruck JT, Pope AL, Ganther HE, Swanson AB, Hafeman DG, Hoekstra WG. Selenium: biochemical role as a component of glatathione peroxidase. Science 1973; 179: 588-90.

16. Buege JA, Aust SD. Microsomal lipid peroxidation. Methods Enzymol 1978; 52: 302-10.

17. Suvarna KS, Layton C BJ. Bancroft's Theory and Practice of Histological Techniques. 8th Ed. Elsevier 2018; 672.

18. Bedossa P, Poynard T. An algorithm for the grading of activity in chronic hepatitis C. Hepatology 1996; 24: 289-93.

19. Duncan T, Valenzuela M. Alzheimer's disease, dementia, and stem cell therapy. Stem Cell Res Ther 2017; 8: 111.

20. Conese M, Piro D, Carbone A, Castellani S, Di Gioia S. Hematopoietic and mesenchymal stem cells for the treatment of chronic respiratory diseases: role of plasticity and heterogeneity. Scientific World Journal 2014; 2014: 859817.

21. Marlicz W, Łoniewski I, Skonieczna-Żydecka K. Stem and progenitor cells in the pathogenesis and treatment of digestive diseases. Adv Exp Med Biol 2019; 1201: 125-57.

22. Ziaei M, Zhang J, Patel D V, McGhee CNJ. Umbilical cord stem cells in the treatment of corneal disease. Surv Ophthalmol 2017; 62: 803-15.

23. Zhang J, Yang A, Wu Y, et al. Stachydrine ameliorates carbon tetrachloride-induced hepatic fibrosis by in- 
hibiting inflammation, oxidative stress and regulating MMPs/TIMPs system in rats. Biomed Pharmacother 2018; 97: 1586-94.

24. Nasir GA, Mohsin S, Khan M, et al. Mesenchymal stem cells and Interleukin- 6 attenuate liver fibrosis in mice. J Transl Med 2013; 11: 78.

25. Negri S, Wang Y, Sono T, et al. Systemic DKK1 neutralization enhances human adipose-derived stem cell mediated bone repair. Stem Cells Transl Med 2021; 10: 610-22.

26. De Luna A, Otahal A, Nehrer S. Mesenchymal stromal cell-derived extracellular vesicles - silver linings for cartilage regeneration? Front Cell Dev Biol 2020; 8: 593386.

27. Yang SH, Park MJ, Yoon IH, et al. Soluble mediators from mesenchymal stem cells suppress T cell proliferation by inducing IL-10. Exp Mol Med 2009; 41: 315-24.

28. Hu C, Zhao L, Duan J, Li L. Strategies to improve the efficiency of mesenchymal stem cell transplantation for reversal of liver fibrosis. J Cell Mol Med 2019; 23: 1657-70.

29. Liu XY, Liu RX, Hou F, et al. Fibronectin expression is critical for liver fibrogenesis in vivo and in vitro. Mol Med Rep 2016; 14: 3669-75.

30. Xu Y, Tang X, Yang M, et al. Interleukin 10 gene-modified bone marrow-derived dendritic cells attenuate liver fibrosis in mice by inducing regulatory $T$ cells and inhibiting the TGF- $\beta /$ Smad signaling pathway. Mediators Inflamm 2019; 2019: 4652596.

31. Wang L, Bai G, Chen F. Human bone marrow mesenchymal stem cells suppress the proliferation of hepatic stellate cells by inhibiting the ubiquitination of p27. Biochem Cell Biol 2017; 95: 628-33.

32. Burger B, Kühl CMC, Candreva T, et al. Oral administration of EPA-rich oil impairs collagen reorganization due to elevated production of IL-10 during skin wound healing in mice. Sci Rep 2019; 9: 9119.

33. Shi J, Li J, Guan H, et al. Anti-fibrotic actions of interleukin-10 against hypertrophic scarring by activation of PI3K/AKT and STAT3 signaling pathways in scar-forming fibroblasts. PLoS One 2014; 9: e98228.

34. Henderson NC, Arnold TD, Katamura Y, et al. Targeting of $\alpha v$ integrin identifies a core molecular pathway that regulates fibrosis in several organs. Nat Med 2013; 19 : 1617-24

35. Koivisto L, Bi J, Häkkinen L, Larjava H. Integrin $\alpha v \beta 6$ : structure, function and role in health and disease. Int J Biochem Cell Biol 2018; 99: 186-96.

36. Costalonga EC, Fanelli C, Garnica MR, Noronha IL. Adipose-derived mesenchymal stem cells modulate fibrosis and inflammation in the peritoneal fibrosis model developed in uremic rats. Stem Cells Int 2020; 2020: 3768718.

37. Benvenuto F, Voci A, Carminati E, et al. Human mesenchymal stem cells target adhesion molecules and receptors involved in T cell extravasation. Stem Cell Res Ther 2015; 6: 245.

38. Popp FC, Slowik P, Eggenhofer E, et al. No contribution of multipotent mesenchymal stromal cells to liver regeneration in a rat model of prolonged hepatic injury. Stem Cells 2007; 25: 639-45.

39. Cejka C, Holan V, Trosan P, Zajicova A, Javorkova E, Cejkova J. The favorable effect of mesenchymal stem cell treatment on the antioxidant protective mechanism in the corneal epithelium and renewal of corneal optical properties changed after alkali burns. Oxid Med Cell Longev 2016; 2016: 5843809. 